\title{
Morphology-controllable synthesis of pyrene nanostructures and its morphology dependence of optical properties
}

Xiujuan Zhang ${ }^{I} \square$ Xiaohong Zhang ${ }^{1, *}$, Wensheng Shi ${ }^{1}$, Xiangmin Meng ${ }^{l}$,

Chunsing Lee $e^{2}$ and Shuittong Lee $e^{1,2 *}$

Supporting information
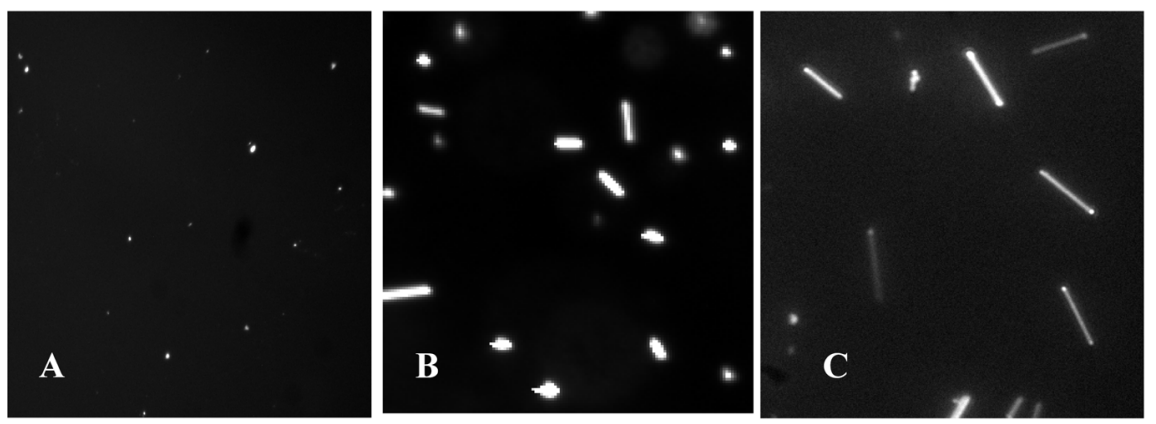

Figure 1 fluorescence microscope images for samples A, B, C respectively

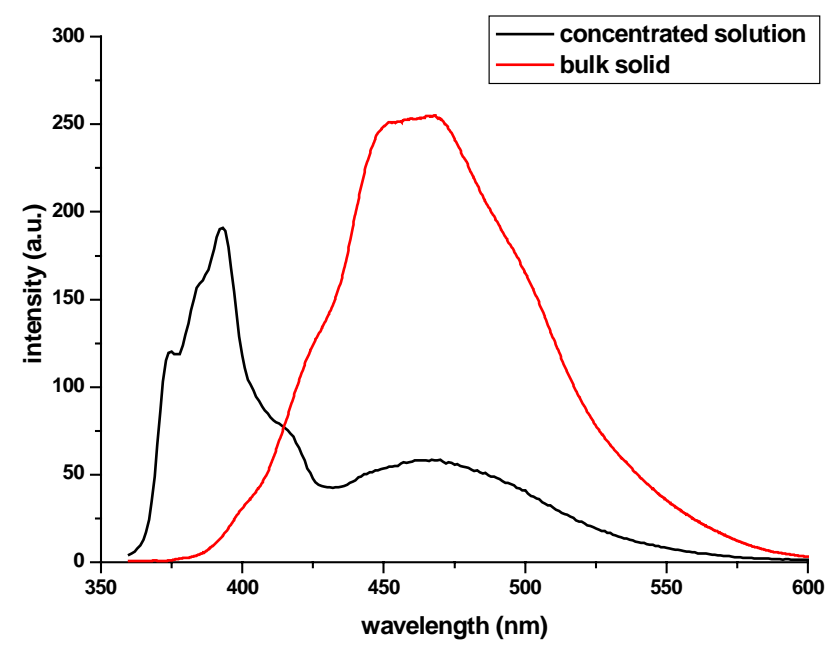


Figure 2 emission spectra for concentrated pyrene solution $\left(1.8 \times 10^{-3} \mathrm{~mol} \mathrm{~L}^{-1}\right)$ and bulk solid. 